\title{
Rhesus monkeys (Macaca mulatta) select Arabic numerals or visual quantities corresponding to a number of sequentially completed maze trials
}

\author{
Emily H. Harris, David A. Washburn, Michael J. Beran, and Rose A. SevciK \\ Georgia State University, Atlanta, Georgia
}

\begin{abstract}
Four number-trained rhesus monkeys were trained to enumerate their sequential responses. After completing a series of computerized maze trials, the monkeys were given a same/different discrimination involving a numerical stimulus (an Arabic numeral or a visual quantity) and the letter $D$. The goal was to choose the numerical stimulus if it matched the number of just-completed maze trials, and to choose the letter $D$ if it did not. There were large individual differences in performance, but one animal performed above $70 \%$ when receiving randomly intermixed series of $1,3,5$, and 9 maze trials. This indicates that the monkey was keeping track of the approximate number of maze trials completed in each series and using that numerical cue to respond during the same/different discrimination.
\end{abstract}

Several different paradigms have been used to investigate numerical ability in animals. These include relative numerousness judgments, in which the animals choose between two or more sets of items on the basis of quantity (e.g., Beran, 2001; Boysen \& Berntson, 1995; Brannon \& Terrace, 2000; Call, 2000; Hauser, Carey, \& Hauser, 2000; Nieder, Freedman, \& Miller, 2002; Roberts \& Mitchell, 1994; Thomas, Fowlkes, \& Vickery, 1980) and tasks in which the absolute number of items is relevant (e.g., Beran \& Rumbaugh, 2001; Boysen \& Berntson, 1989; Capaldi \& Miller, 1988; Davis, 1984; Emmerton, 1998; Matsuzawa, 1985; Murofushi, 1997; Pepperberg, 1994; Xia, Emmerton, Siemann, \& Delius, 2001). The present study uses a paradigm in which the "to-be-enumerated" items are sequential events rather than visible items, because we are interested in whether number-trained rhesus monkeys can match their own sequential responses with an Arabic numeral or visual dot quantity.

One of the many important aspects of human numerical competence involves the ability to keep track of sequentially presented items or events and to provide a numerical label corresponding to the cardinal value of the set. For example, adult humans asked to keep track of the number of traffic lights they pass on their way to work each morning would probably be able to provide the correct number. Several researchers have used the sequential presentation of items or events to investigate numerical ability and serial learning in rats (e.g., Burns \& Criddle, 2001; Burns, Johnson, Harris, Kinney, \& Wright, 2004; Capaldi $\&$ Miller, 2004). In one such study, Davis and Bradford (1986) trained rats to enter either the third or fourth tunnel in a series of six tunnels. The configuration of the tunnels and distance between them varied from trial to trial, so the only available cue was the number of previously encountered tunnels.

Capaldi and Miller (1988) trained rats with a three-trial series of maze runs consisting of two reinforced trials followed by a nonreinforced trial (RRN) and a four-trial (NRRN) series beginning and ending with a nonreinforced trial. The rats quickly developed a pattern of running more slowly on the terminal nonreinforced trial of each series than on the other, reinforced trials. This indicates that they were keeping track of the number of completed trials and predicting when the nonreinforced trial would occur. Burns, Goettl, and Burt (1995) systematically varied the intertrial intervals in a series of runway trials and concluded that the slower running times observed on the terminal nonreinforced trials could not be explained by rhythmic cues, as had been suggested by Davis and Pérusse (1988).

A recent study from our laboratory focused on the ability of four number-trained rhesus monkeys, including those in the present study, to use an Arabic numeral cue to predict when a nonreinforced event would occur (Harris $\&$ Washburn, 2005). The monkeys were presented with a computerized task consisting of three reinforced maze trials followed by one nonreinforced trial (RRRN). The goal of the maze was an Arabic numeral 3, which corresponded to the number of reinforced trials in the series. Two of the monkeys eventually developed a "slow, fast, faster, slow" pattern similar to that of the rats in the Capaldi and Miller (1988) study. Judging by the slow running time on the terminal nonreinforced trial of the series, the monkeys had been anticipating the nonreinforced trial. The other two 
monkeys performed gradually slower on each trial in the series, which made it difficult to speculate on their ability to predict the nonreinforced trial.

Two of the monkeys then were given probe series of the numerals 2 and 4 , and the remaining 2 monkeys were given probe series of the numerals 2 through 8 . These probe series were randomly intermixed with the familiar numeral 3 series. As was true during training, the Arabic numeral displayed in the maze corresponded to the number of reinforced trials that would occur before one nonreinforced trial. For instance, a numeral 2 indicated that it was an RRN series.

The monkeys receiving the probe series 2 and 4 showed generalization to the new numerals and developed a pattern of performing more slowly on the nonreinforced trial than on the reinforced trials before it, indicating the use of the changing target numeral to anticipate the nonreinforced trial. The monkeys receiving probe series of the numerals 2 through 8 did not use the changing numerals to predict precisely when the nonreinforced trial would occur in each series, but they did incorporate numerical cues into their performance strategy. They responded differentially to the targets by running faster overall on series with higher target numerals. One explanation for this result is that the monkeys recognized that a higher target numeral indicated more reinforced trials before the one nonreinforced trial. This may have motivated the monkeys to perform faster overall on those series.

Although not all of the monkeys in the Harris and Washburn (2005) study used the target numbers in the way anticipated, there was little motivation for the monkeys to keep track of the absolute number of trials. The reinforcement pattern remained the same, no matter what strategy the monkeys used to perform the task. During training, for instance, the monkeys always received three reinforced trials followed by one nonreinforced trial, regardless of how quickly they completed each maze trial. In addition, the monkeys performed thousands of trials a day on this task and other tasks, so a few nonreinforced trials were probably not very salient.

In the present study, the monkeys were required to compare the number of maze trials they had just completed with two choice options, and they were reinforced only when they made a correct response. This would increase motivation to perform at high levels because of the time invested in each series of maze trials. The current study is unique in that it not only tests the ability of numbertrained monkeys to keep track of sequential events, but also tests their ability to compare numerical labels for cardinal values to sequentially completed responses that must be enumerated. Although chimpanzees have demonstrated an ability to label a visible quantity of items with an Arabic numeral (e.g., Biro \& Matsuzawa, 2001; Boysen \& Berntson, 1989; Matsuzawa, 1985; Murofushi, 1997; Tomonaga \& Matsuzawa, 2002), this ability in rhesus monkeys has never been demonstrated with simultaneously visible items or sequentially completed events.

In this experiment, the monkeys received series of 1,2, 3,5 , or 9 computerized maze trials, followed by two response options. One option was a numerical stimulus (ei- ther an Arabic numeral or a dot array) that either matched or differed from the number of maze trials that had been completed. The other option was a letter $D$, which represented "different" from the number of maze trials in the syntax of the computer program. We were interested in whether the monkeys could learn to choose the numerical stimulus when it matched the number of just-completed maze trials, or to choose the $D$ when the numerical option did not match the number of just-completed maze trials. Because these animals had previously been trained to use Arabic numerals in quantity judgment tasks (e.g., Washburn \& Rumbaugh, 1991), we also wanted to investigate any potential differences in performance as a function of the form that the numerical response option took (as either a numeral or a dot quantity). Given the previous manner in which the monkeys used numerals, we predicted that sequentially enumerated sets might be more easily represented as visual quantities, and that performance might be higher when the numerical response option took the form of a visual dot quantity. However, if Arabic numerals represented abstract quantities for the monkeys, perhaps those stimuli also could be used appropriately within this task.

\section{METHOD}

\section{Subjects}

Four male rhesus monkeys (Macaca mulatta; Willie, Gale, Lou, and Hank) participated in this study. Their ages were 18,20,10, and 18 years, respectively. The monkeys were housed individually at the Language Research Center of Georgia State University according to federal animal housing standards. They were not deprived of food or water during this study.

All of these monkeys had participated in previous studies that required them to make relative numerousness and ordinal judgments using Arabic numerals and visual dot displays (e.g., Gulledge, 1999; Washburn \& Rumbaugh, 1991). All except Hank also had experience in performing series of maze trials (Harris \& Washburn, 2005). In addition, all four monkeys had participated in computerized joystick tasks related to various areas of cognitive research (e.g., Smith, Shields, \& Washburn, 2003; Washburn \& Gulledge, 2002; Washburn \& Rumbaugh, 1997), including same/different judgments similar to those used in the current task, but with nonnumerical stimuli.

\section{Design and Procedure}

The monkeys were tested in their home cages using the Language Research Center Computerized Test System (see Rumbaugh, Richardson, Washburn, Savage-Rumbaugh, \& Hopkins, 1989, for a description), which consists of a joystick attached to a computer and color monitor. The monkeys moved the joystick to control the movement of the cursor on the screen. The computer program recorded the target number along with the duration of each maze trial, the choices presented, and the accuracy and response time for each stimulus choice.

The computerized display consisted of a black $\mathrm{H}$-shaped maze on a white background (Figure 1). The goal stimulus in the maze was a green rectangle appearing in one of four corners of the maze. The computer program randomly selected the corner on each trial. The monkey initiated the start of each trial by moving the joystick. At the beginning of each trial, the cursor appeared in the middle of the maze, and the monkey was required to move the cursor through the maze to the goal in order to complete a trial successfully.

Each series consisted of 1, 2, 3, 5, or 9 maze trials. The monkeys started with series of 1 and 9 maze trials, and additional series were added as the monkeys reached an accuracy criterion (see below). 


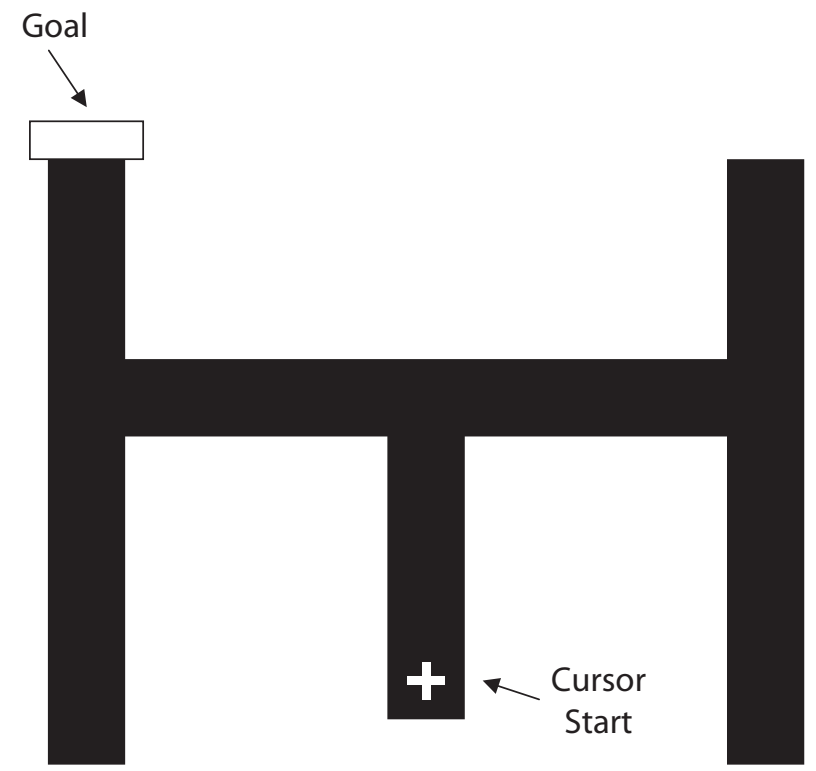

Figure 1. The maze display used during the series. The goal was colored green.

Once contact was made with the square at the end of the maze, the cursor returned to the middle of the maze and a new trial began with a new, randomly selected goal location. This meant that the animals could not use learned motor sequences for different numbers of maze trials because of the high number of variations of placement of the goals during each series. Completion of individual trials was not reinforced.

The monkeys involved in this study had been trained previously to pick the stimulus displaying the largest numerosity from an array. In contrast, this study required the monkeys to choose only numerical stimuli that matched the number of maze trials in a series. To avoid the monkeys' bias toward picking larger numbers, a same/different judgment was used instead of a matching-to-sample procedure.

Upon completion of all the maze trials in a series, the maze disappeared and two different stimulus choices immediately appeared on the screen. One choice (the numerical choice) was an Arabic numeral or visual quantity display and the other was the letter $D$, for "different." The $D$ was white on a black background and was sized approximately $3 \mathrm{~cm} \times 3 \mathrm{~cm}$. The Arabic numerals also were white on a black background, and all were approximately $3 \mathrm{~cm} \times 3 \mathrm{~cm}$. The visual quantity display used randomly chosen white polygons of different sizes (hereafter referred to as "dots") on a black background. These polygons were unlike the round dot stimuli previously used with the monkeys in other numerical tasks. There were 10 different polygons used in the study, varying in approximate size from .5 to $.75 \mathrm{~cm}$. Each polygon in the visual array was placed in a random location within an invisible $5 \times 5$ matrix. Both the Arabic numerals and the dots were presented within a white $5 \mathrm{~cm} \times 5 \mathrm{~cm}$ square.

The numerical stimulus presented during the labeling phase always corresponded to a possible number of maze trials from that test session. For example, when the monkeys were receiving only 1 or 9 maze trials in a series, the stimuli 1 and 9 (presented as numerals or dot quantities) were the only numerical stimuli choices used in the labeling phase. Within this constraint, the value of the Arabic numeral or number of dots was selected randomly by the computer program. The computer program randomly assigned the numerical stimulus to appear on the left or right side of the screen with the constraint that no more than four consecutive series could have the numerical stimulus displayed on the same side. The $D$ appeared on the opposite side of the screen from the numeral or dot stimulus (Figure 2).
The type of numerical stimulus (numeral or dot quantity) that was presented also varied randomly from series to series, with the constraint that no more than four consecutive series could have the same type of stimulus. This was to ensure that one type of stimulus was not presented much more often than another, which would have caused the monkeys to form a bias toward the particular numeral or visual display, on the basis of the number of trials received.

If the numeral or visual dot quantity displayed during the labeling phase matched the number of maze trials in that series, the goal for the monkey was to move the cursor from the middle of the screen and make contact with that numerical stimulus. If the numeral or number of dots did not match the number of maze trials in the series, the goal was to move the cursor and make contact with the letter $D$.

Before each labeling phase, the computer program randomly determined whether the correct choice would be "same" or "different." Therefore, the correct choice was the dot quantity or numeral for approximately half of the discriminations and the letter $D$ for the other half. This ensured that the monkeys needed to use the changing number of maze trials in order to be reinforced at a greater-thanchance level.

Correct responses during the labeling phase were rewarded with sound feedback and the automatic delivery of $94-\mathrm{mg}$ fruit-flavored pellets. The number of pellets delivered corresponded to the number of maze trials in that series. For instance, a correct response after a two-trial series was rewarded with two pellets. Incorrect responses resulted in a 15 -sec time-out and a negative buzzing sound. After the monkeys had completed the labeling phase of a series, a new series of maze trials began. To prevent the monkeys from developing a bias toward the $D$ or the numerical stimuli, incorrect series were repeated until the monkeys made the correct choices.

The monkeys had continuous access to the task for several hours a day, several days a week. At the start of testing, they were presented with randomly intermixed series consisting of 1 or 9 maze trials and discriminations involving only the numerical stimulus 1 or 9 and the $D$. (We started the maze trials with the extreme values of 1 and 9 to aid the monkeys in conceptually connecting the maze trials and the discriminations.) Trials were administered in 100-series blocks. One additional numerosity $(5,3$, or 2 , in that order) was added each time the monkeys reached a performance level of $70 \%$ or better over the three most recent blocks. These additional numerosities were randomly intermixed with the familiar numerosities.

The additional numerosities $(5,3$, and 2$)$ were all chosen to facilitate learning of the task. The numeral 5 was chosen to take ad-

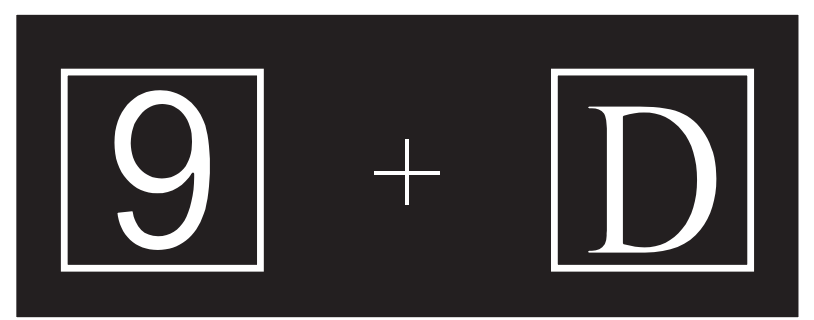

OR

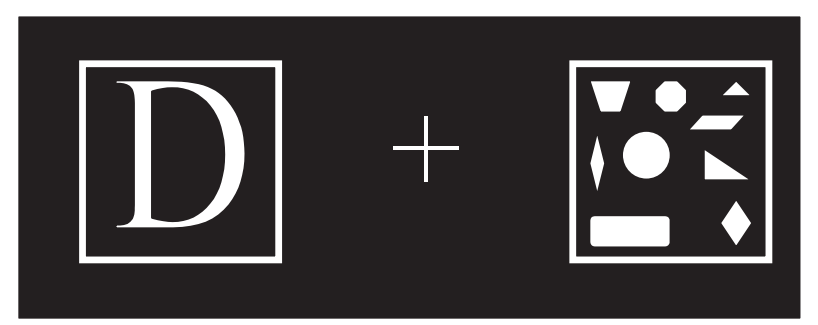

Figure 2. Example of display used during the labeling phase of a series. The "+" in the center of each figure is the cursor. 
vantage of the distance effect, which suggests that discriminations are easier when two numbers are farther apart. The numerals 2 and 3 were chosen to take advantage of the magnitude effect, which suggests that when distance is held constant, discriminations are easier with smaller numbers compared to larger ones (Moyer \& Landauer, 1967).

Testing ended for each monkey when it failed to reach criterion after 30 blocks (3,000 series) with a given set of randomly intermixed numerosities. Additional numerosities were not used because none of the monkeys achieved the accuracy criterion with the numerosities $1,2,3,5$, and 9 .

\section{Analyses}

As stated previously, when a monkey gave an incorrect response, the series was repeated until a correct response was given. The first response was included in analyses, and all correction series were excluded.

The monkeys were not restrained during this task, so they occasionally took a break to rest, eat, drink water, utilize another enrichment device, or engage in social behavior. This could result in unrealistically long trial times, and it caused the mean times to be much greater than the medians. All series in which a maze trial lasted longer than $10 \mathrm{sec}$ were excluded from analyses, because this duration was about three times that of the typical maze trial. Across the four monkeys, this resulted in the exclusion of an average of $3.73 \%$ of the series. To ensure that the exclusion of these series was justified, the medians for the maze trials were analyzed. The medians were comparable to the means obtained when excluding these series, so the 10 -sec trial limit was used in all subsequent analyses.

\section{RESULTS}

Table 1 shows the number of blocks ( 100 series each) required by each monkey to reach the $70 \%$ accuracy criterion after each new numerosity was added. All of the monkeys achieved the accuracy criterion when presented with only the numerosities 1 and 9; three of the four monkeys reached criterion with the numerosities 1,5 , and 9; and one monkey reached criterion with the numerosities 1,3 , 5 , and 9. However, none of the monkeys was able to reach criterion when presented with the numerosities 1, 2, 3, 5, and 9. The number of trials required for the monkeys to reach criterion with two numerosities was not a good predictor of how well they performed overall on this task. For example, Hank required more trials than any other monkey to reach criterion with two numerals, but he was the only monkey to achieve criterion with four numerosities.

Only data from the last 3,000 series performed by the monkeys were used in subsequent analyses. These trials were chosen because they contain the greatest range of numerosities for each monkey and therefore provide the

Table 1

The Number of Blocks (100 Series per Block) Required for Each Monkey to Reach the $\mathbf{7 0 \%}$ Accuracy Criterion on Each Set of Numerosities

\begin{tabular}{lrcc}
\hline & 1,9 & $1,5,9$ & $1,3,5,9$ \\
\hline Willie & 10 & & \\
Gale & 3 & 3 & \\
Lou & 4 & 5 & 10 \\
Hank & 13 & 4 & 10 \\
\hline
\end{tabular}

Note-Empty cells indicate that the monkey did not reach criterion for that set of numerosities. greatest opportunity for analyses relevant to the experimental questions and hypotheses. Although the monkeys did not achieve the $70 \%$ accuracy criterion for these trials, they had achieved the accuracy criterion for the previous set of numbers. Therefore, at the start of the 3,000 series, the monkeys were already performing at greaterthan-chance levels with every numerosity except the most recent addition.

To assess possible practice effects over the course of the last 3,000 series (30 blocks), correlation coefficients were computed for each monkey to determine whether accuracy increased or decreased as block number increased. No significant correlations ( $p<.05$, two-tailed) were found for any of the monkeys (Willie, $r=.11$; Lou, $r=$ .06; Gale, $r=.24$; Hank, $r=-.18$ ), indicating that the performance of these monkeys did not change significantly. Thus, these 3,000 series represent full, mature performance on the task.

Figure 3 shows the percentage accuracy for each number of maze trials and each stimulus type. Although Gale and Willie both show significantly higher accuracy $(p<$ .05 ) when presented with numerical stimuli in the form of Arabic numerals for at least one number of maze trials, there is no consistent pattern to indicate a meaningful interaction. For example, Gale and Willie were not consistently more accurate on numeral trials than on dot quantity trials for series with low, high, or intermediate numbers of maze trials. Lou showed the opposite pattern of performance for series with one maze trial, which provides further evidence that there is no meaningful interaction between stimulus type and target number.

A two-way ANOVA of the effect of stimulus and trial type on accuracy was performed, using data from all four monkeys. Stimulus type refers to the form in which the numerosity was presented (numeral or dot quantity) and trial type refers to the correct response required for that trial (the numerical stimulus or the $D$ ). Although power was low, this analysis yielded no significant differences [stimulus type, $F(1,3)=3.33, p=.17, \eta^{2}=.53$, observed power $=.25$; trial type, $F(1,3)=1.63, p=.29, \eta^{2}=.35$, observed power $=.15$; stimulus type $\times$ trial type interaction, $F(1,3)=1.20, p=.35, \eta^{2}=.29$, observed power $=$ .12]. Descriptive statistics also indicate that there was no bias toward one type of stimulus or trial type [dots with a numerical stimulus response $M(S D)=62.52 \%(8.50 \%)$; dots with a $D$ response $M(S D)=63.03 \%(8.87 \%)$; numerals with a numerical stimulus response $M(S D)=72.55 \%$ (3.36\%); numerals with a $D$ response $M(S D)=62.77 \%$ (3.81\%)]. Given this result of no difference in performance as a function of the form of the numerical stimulus presented at the labeling phase of the series, trial type and stimulus type were combined for all subsequent analyses, unless otherwise noted.

To test for a distance effect, the accuracy of all four monkeys was regressed on the numerical difference between the number of maze trials completed and the numerical stimulus that was presented during the labeling phase. The regression analysis revealed that accuracy was positively associated with the difference $[F(1,15)=$ 

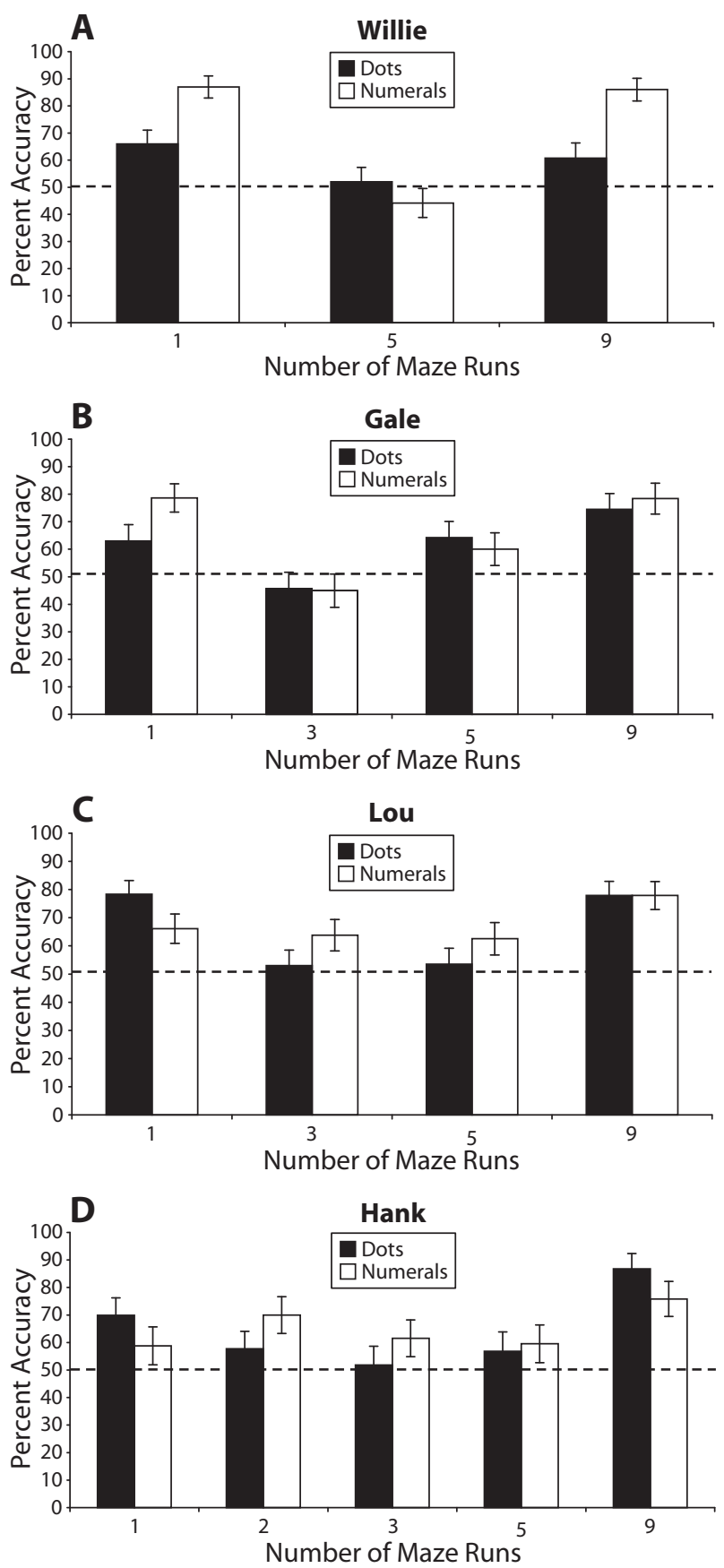

Figure 3. Percent accuracy for each type of stimulus and each number of maze runs. The dotted line denotes chance level. Error bars denote $95 \%$ confidence intervals.

$\left.48.55, p<.05, R^{2}=.76\right]$. It must be noted, however, that practice effects may have contributed to this correlation. The monkeys had the most practice with the numerosities 1 and 9 , which are also the two numerosities farthest apart in distance. The magnitude of the numerosities involved could also have affected the correlation. To take into account the magnitude of the numerosities as well as their numerical distance, accuracy was regressed on the ratio of the smaller numerosity to the larger numerosity used in each series. For example, if a monkey completed one maze trial and was presented with the numeral 5 during the labeling phase, the ratio would equal 0.2. This regression analysis revealed that accuracy was significantly associated with ratio $\left[F(1,20)=63.39, p<.01, R^{2}=.76\right]$. This effect is illustrated in Figure 4.

To determine whether the monkeys were using the combined duration of the maze trials, instead of their numerical value to perform this task, data were analyzed from all of the trials on which the monkeys chose the numerical stimulus during the discrimination trial. We chose to look at this question post hoc rather than controlling for duration experimentally, because manipulating the duration of the trial would have caused other factors, such as rate of maze completion, to covary with the number of trials in a series. If the monkeys were using duration as a cue to this task, incorrect trials in which the monkeys chose a numerosity smaller than the number of maze trials performed should have occurred when the total duration of the maze trials was shorter than usual when they responded correctly for a given number of maze trials (i.e., incorrect maze trial duration $<$ mean correct maze trial duration). In contrast, incorrect trials in which the monkeys chose a numerosity larger than the number of maze trials performed should have occurred when the total duration of the maze trials was larger than it usually was for correct trials (i.e., incorrect maze trial duration > mean correct trial duration).

For each monkey, a one-way ANOVA assessing the effects of trial type on the total duration of the maze trials was performed for each number of maze runs. The types of trial were categorized as those in which the monkey chose a numerical stimulus larger than the number of maze runs, those in which the monkey chose a numerical stimulus smaller than the number of maze runs, and those in which the monkey correctly chose the numerical stimulus. The only significant effect was found for Willie. For trials in which he performed only one maze run, his maze trial durations were significantly shorter on trials in which he chose a numerical stimulus larger than the number of maze trials compared with trials in which he correctly chose the numerical stimulus $[F(1,405)=4.49$, $\left.p<.05, \eta^{2}=.01\right]$. This effect is opposite to what was predicted for a strategy involving duration as a cue to the correct response. Thus, none of the monkeys used differences in maze completion duration as the cue for which stimulus to select during the labeling phase.

\section{DISCUSSION}

During the course of this study, the monkeys learned to label a series of sequentially completed maze trials with the corresponding Arabic numeral or visual dot quantity (or a $D$ if the numerical option was not equal). All of the monkeys learned to match randomly intermixed series of 1 or 9 maze trials with the correct Arabic numeral or visual quantity when tested with a same/different discrimination. This provides evidence that the monkeys understood the task on some level and conceptually connected the maze series with the same/different discriminations. 


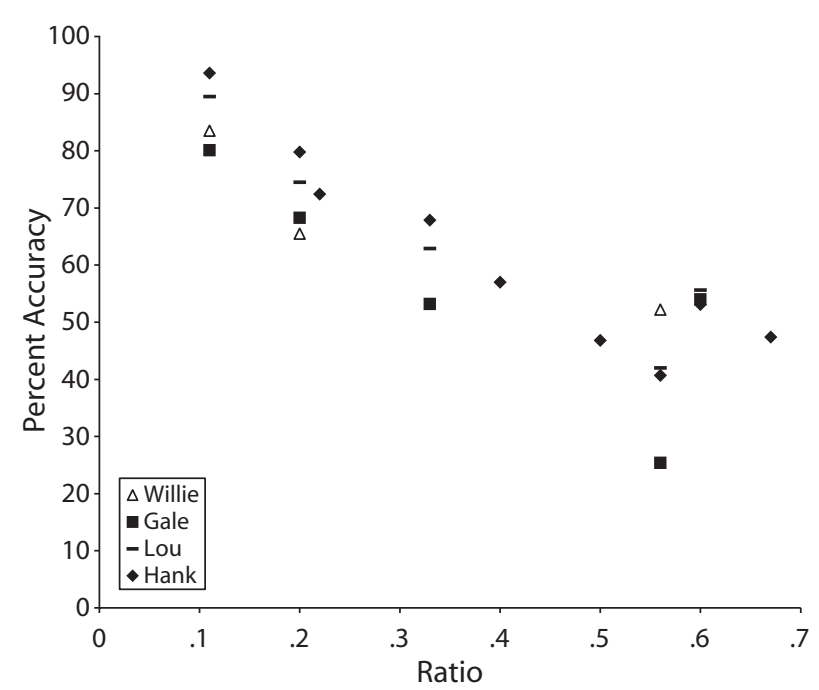

Figure 4. Accuracy as a function of the ratio of the smaller numerosity to the larger numerosity.

This part of the task, however, could be performed by representing the number of maze trials simply as "few" and "many" (or "one and "many"), without representing the number of maze runs as a specific quantity. In fact, Willie seems to have used one of those strategies throughout this experiment. Willie achieved an accuracy level of $70 \%$ fairly quickly when presented with series of one and nine maze trials, but he did not achieve the accuracy criterion when the numerosity 5 was added. His pattern of errors revealed that after performing one maze run he almost never chose the numerosities 5 or 9 , but after performing five maze runs he was more likely to choose 9 than 1 , and after performing nine maze runs he was more likely to choose 5 than 1 . This indicates confusion between the numerosities 5 and 9 that was not present for the numerosity 1 .

Two of the monkeys participating in this study achieved accuracies greater than $70 \%$ for the numerosities 1,5 , and 9 within the first 500 presentations, but they did not reach criterion when the numerosity 3 was added to the experimental set. Their ability to perform the task with three numerosities indicates that their representation of the maze runs went beyond a simple representation of "one" and "many."

The fourth monkey in the study, Hank, performed the task with the numerosities $1,3,5$, and 9 , but failed to achieve the $70 \%$ accuracy criterion after the numerosity 2 was added to the set. It is important to note that these numerosities were randomly intermixed, so Hank never received blocks of series containing only one numerosity. Rather, each new series of maze trials could consist of any of the numerosities in the set.

The monkeys were reinforced for correct choices with a number of pellets equal to the number of maze trials performed in the just-completed series as a motivation to complete the longer series. This did cause a slight highnumber choice bias on pairs of trials in which the distance and magnitude of the two numerosities were the same.
For instance, the monkeys were less accurate on trials in which they ran one maze trial and were presented with the numerosity 9 than they were on trials in which they ran nine maze trials and were presented with the numerosity 1 (an average accuracy of 79\% in the former case and $95 \%$ in the latter). However, this bias did not prevent any of the monkeys from exceeding $70 \%$ accuracy for trials in which they performed one maze run and were presented with the tempting numerosity 9 . The bias was even less pronounced for the numerosities 2, 3, and 5 .

There is some evidence that the monkeys were using an approximate and variable representation of the number of maze runs to perform this task. Accuracy increased as a function of distance between the number of maze trials and the numerosity presented during the discrimination. Accuracy also decreased as a function of the ratio of the smaller numerosity to the larger numerosity used in each series, as predicted by Weber's law. Although a greater amount of practice with the numerosities 1 and 9 as compared to other numerosity pairs may have contributed to this correlation, a distance effect and adherence to Weber's law would occur if the monkeys' numerical representations were composed of inexact magnitudes. This is because inexact magnitudes would be more difficult to compare when the numerosities were close in distance and/or large in magnitude (Dehaene, 1997; Gallistel \& Gelman, 2000).

The monkeys' error patterns were not related to the amount of time they spent on the maze trials in each series. The monkeys did not tend to choose numerosities that were higher than the correct choice after spending more time than usual on a particular series; therefore, they were not using duration alone as a cue to performing this task.

It is interesting that the monkeys performed equally well when the numerical stimulus was in Arabic numeral or visual dot quantity form. Although the visual quantities provide more inherent numerical information than the numerals, the monkeys have had a variety of testing experiences involving Arabic numerals. Their ability to match a series of maze trials to either a visual quantity or an Arabic numeral indicates flexibility in their performance strategy.

The ability of the monkeys to perform this task is impressive, due to the working memory demands and the absence of perceptual cues, such as surface area or density, to aid in the formation of their numerical representations. The monkeys were required to form a representation of a series of events, which lacked standard perceptual features, to update this representation throughout the series of maze trials, and to keep this representation in working memory while they chose the appropriate stimulus during the same/different discrimination. Previous studies have found that nonhuman primates are capable of representing, combining, and comparing nonvisible, sequentially presented sets of items (e.g., Beran, 2001; Call, 2000; Hauser et al., 2000). This experiment provides strong evidence that monkeys can enumerate, albeit approximately, their own sequential responses, and can match the number of responses with the corresponding Arabic numeral or visual quantity. 


\section{AUTHOR NOTE}

This research was supported by a Grant HD-38051 from the National Institute of Child Health and Human Development to the Language Research Center of Georgia State University, and by a Rumbaugh Fellowship to E.H.H. Portions of this research were submitted to Georgia State University by E.H.H. as partial fulfillment for the degree of Master of Arts. The authors thank Eric J. Vanman for his thoughts and comments during this project. All applicable federal, disciplinary, and institutional rules and regulations regarding animal care and use have been followed in the care and testing of the monkeys. Address all correspondence concerning this article to E. H. Harris, Language Research Center, Georgia State University, 3401 Panthersville Road, Decatur, GA 30034 (e-mail: eharris11@gsu.edu).

\section{REFERENCES}

Beran, M. J. (2001). Summation and numerousness judgments of sequentially presented sets of items by chimpanzees (Pan troglodytes). Journal of Comparative Psychology, 115, 181-191.

BeRAN, M. J., \& RUMBaUGH, D. M. (2001). "Constructive" enumeration by chimpanzees (Pan troglodytes) on a computerized task. Animal Cognition, 4, 81-89.

Biro, D., \& MATSUZAWA, T. (2001). Use of numerical symbols by the chimpanzee (Pan troglodytes): Cardinals, ordinals, and the introduction of zero. Animal Cognition, 4, 193-199.

Boysen, S. T., \& BernTSON, G. G. (1989). Numerical competence in a chimpanzee (Pan troglodytes). Journal of Comparative Psychology, 103, 23-31.

BoYsen, S. T., \& Berntson, G. G. (1995). Responses to quantity: Perceptual versus cognitive mechanisms in chimpanzees (Pan troglodytes). Journal of Experimental Psychology: Animal Behavior Processes, $\overline{21,82-86 .}$

BranNon, E. M., \& Terrace, H. S. (2000). Representation of the numerosities 1-9 by rhesus macaques (Macaca mulatta). Journal of Experimental Psychology: Animal Behavior Processes, 26, 31-49.

BURNS, R. A., \& CRIDDLE, C. R. (2001). Retention of ordinal position information with limited and extended serial training. Psychological Record, 51, 445-452.

BuRNS, R. A., GOETTL, M. E., \& Burt, S. T. (1995). Numerical discrimination with arrhythmic serial presentations. Psychological Record, 45, 95-104.

Burns, R. A., Johnson, K. S., Harris, B. A., Kinney, B. A., \& Wright, S. E. (2004). Functional cues for position learning effects in animals. Psychological Record, 54, 233-254.

CALL, J. (2000). Estimating and operating on discrete quantities in orangutans (Pongo pygmaeus). Journal of Comparative Psychology, 114, 136-147.

CAPALDI, E. J., \& MiLler, D. J. (1988). Counting in rats: Its functional significance and the independent cognitive processes that constitute it. Journal of Experimental Psychology: Animal Behavior Processes, 14, 3-17.

CAPAldi, E. J., \& Miller, D. J. (2004). Serial learning in rats: A test of three hypotheses. Learning \& Motivation, 35, 71-81.

Davis, H. (1984). Discrimination of the number three by a raccoon (Procyon lotor). Animal Learning \& Behavior, 12, 409-413.

DaVIS, H., \& BRAdFord, S. A. (1986). Counting behavior by rats in a simulated natural environment. Ethology, 73, 265-280.

DAvis, H., \& PÉRUSSE, R. (1988). Numerical competence in animals: Definitional issues, current evidence, and a new research agenda. $\mathrm{Be}$ havioral \& Brain Sciences, 11, 561-615.
Dehaene, S. (1997). The number sense: How the mind creates mathematics. New York: Oxford University Press.

EMmerton, J. (1998). Numerosity differences and effects of stimulus density on pigeons' discrimination performance. Animal Learning \& Behavior, 26, 243-256.

GALLISTEL, C. R., \& GELMAN, R. (2000). Non-verbal numerical cognition: From reals to integers. Trends in Cognitive Sciences, 4, 59-65.

Gulledge, J. P. (1999). Judgments of dot arrays and Arabic numerals by rhesus macaques: Evidence of concept of number. Unpublished master's thesis, Georgia State University, Atlanta.

HARRIS, E. H., \& WASHBURN, D. A. (2005). Macaques' (Macaca mulatta) use of numerical cues in maze trials. Animal Cognition, 8, 190-199.

Hauser, M. D., Carey, S., \& Hauser, L. B. (2000). Spontaneous number representation in semi-free-ranging rhesus monkeys. Proceedings of the Royal Society of London: Series B, 267, 829-833.

MatsuZaWa, T. (1985). Use of numbers by a chimpanzee. Nature, $\mathbf{3 1 5}$, 57-59.

MOYER, R. S., \& LANDAUER, T. K. (1967). Time required for judgments of numerical inequality. Nature, 215, 1519-1520.

Murofushi, K. (1997). Numerical matching behavior by a chimpanzee (Pan troglodytes): Subitizing and analogue magnitude estimation. Japanese Psychological Research, 39, 140-153.

NiEdER, A., Freedman, D. J., \& Miller, E. K. (2002). Representation of the quantity of visual items in the primate prefrontal cortex. Science, 297, 1708-1711.

PePPERBERG, I. M. (1994). Numerical competence in an African grey parrot (Psittacus erithacus). Journal of Comparative Psychology, 108, 36-44.

ROBERTS, W. A., \& Mitchell, S. (1994). Can a pigeon simultaneously process temporal and numerical information? Journal of Experimental Psychology: Animal Behavior Processes, 20, 66-78.

Rumbaugh, D. M., Richardson, W. K., Washburn, D. A., SavageRumbaugh, E. S., \& Hopkins, W. D. (1989). Rhesus monkeys (Macaca mulatta), video tasks, and implications for stimulus-response spatial contiguity. Journal of Comparative Psychology, 103, 32-38.

Smith, J. D., ShieldS, W. E., \& Washburn, D. A. (2003). The comparative psychology of uncertainty monitoring and metacognition. Behavioral \& Brain Sciences, 26, 317-373.

THOMAS, R. K., FowlKeS, D., \& VICKERY, J. D. (1980). Conceptual numerousness judgments by squirrel monkeys. American Journal of Psychology, 93, 247-257.

ToMONAGA, M., \& MATSUZAWA, T. (2002). Enumeration of briefly presented items by the chimpanzee (Pan troglodytes) and humans (Homo sapiens). Animal Learning \& Behavior, 30, 143-157.

Washburn, D. A., \& Gulledge, J. P. (2002). A species difference in visuospatial working memory in human adults and rhesus monkeys: The concentration game. International Journal of Comparative Psychology, 15, 288-302.

Washburn, D. A., \& Rumbaugh, D. M. (1991). Ordinal judgments of numerical symbols by macaques (Macaca mulatta). Psychological Science, 2, 190-193.

WAShburn, D. A., \& Rumbaugh, D. M. (1997). Faster is smarter, so why are we slower? A comparative perspective on intelligence and processing speed. American Psychologist, 52, 1147-1148.

Xia, L., Emmerton, J., Siemann, M., \& Delius, J. D. (2001). Pigeons (Columba livia) learn to link numerosities with symbols. Journal of Comparative Psychology, 115, 83-91.

(Manuscript received May 15, 2006; revision accepted for publication July 31, 2006.) 